\title{
Canine DVL2 variant contributes to brachycephalic phenotype and caudal vertebral anomalies
}

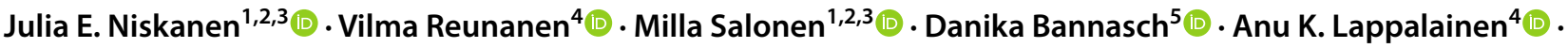 \\ Hannes Lohi ${ }^{1,2,3}$ (1) Marjo K. Hytönen ${ }^{1,2,3}$
}

Received: 16 December 2020 / Accepted: 2 February 2021 / Published online: 18 February 2021

(c) The Author(s) 2021

\begin{abstract}
A frameshift deletion variant in the Wnt pathway gene dishevelled 2 (DVL2) is associated with a truncated, kinked tail ("screw tail") in English Bulldogs, French Bulldogs and Boston Terriers. These breeds are also characterized by distinctive morphological traits, including a wide head, flat face and short-limbed dwarfism, which are characteristic of Robinow syndrome in humans, caused by defects in genes such as $D V L 1$ and $D V L 3$. Based on these phenotypic and genetic similarities, it has previously been hypothesized that the canine $D V L 2$ variant results in a syndromic phenotype called the Robinow-like syndrome. In our study, we investigated the distribution of the DVL2 variant in 1954 dogs from 15 breeds, identifying breeds with allele variation and enabling the dissection of the genotype-phenotype correlation for the first time. With CT examinations in American Staffordshire Terriers, we confirmed that the $D V L 2$ allele is associated with caudal vertebral malformations and a brachycephalic phenotype. We also hypothesize that the variant may be linked to additional health conditions, including brachycephalic obstructive airway syndrome and congenital heart defects. Altogether, our study strengthens the role of $D V L 2$ as one of the contributors to the "bulldog type" morphology and features on the spectrum of human Robinow syndrome.
\end{abstract}

\section{Introduction}

The domestic dog (Canis lupus familiaris) exhibits tremendous morphological variety due to breed propagation. Recently, Mansour et al. (2018) showed that caudal vertebral malformations, or a shortened and kinked tail (also referred to as a "screw tail"), are a consequence of a frameshift deletion variant in the $D V L 2$ (dishevelled segment polarity protein 2) gene. Screw tail is a distinctive trait in English

\footnotetext{
Hannes Lohi

hannes.lohi@helsinki.fi

Marjo K. Hytönen

marjo.hytonen@helsinki.fi

1 Department of Medical and Clinical Genetics, University of Helsinki, Helsinki, Finland

2 Folkhälsan Research Center, Helsinki, Finland

3 Department of Veterinary Biosciences, University of Helsinki, Helsinki, Finland

4 Department of Equine and Small Animal Medicine, University of Helsinki, Helsinki, Finland

5 Department of Population Health and Reproduction, University of California Davis, Davis, CA 95616, USA
}

Bulldogs, French Bulldogs, and Boston Terriers. These breeds are also characterized by other vertebral anomalies, brachycephalic skull morphology, widely set eyes, and short stature. Similar clinical signs are seen in Robinow syndrome in humans, a hereditary disorder caused by gene defects in the Wnt signaling pathway, including DVL1, DVL3, ROR2, WNT5A, FZD2 and NXN (White et al. 2018). Robinow syndrome is characterized by distinctive facial features, e.g., prominent forehead, widely spaced eyes and a flat nasal bridge; mesomelic limb shortening; and variable cardiac, oral and urogenital anomalies (Soman and Lingappa 2015). Based on the genetic and clinical similarities between Robinow patients and these breeds, Mansour et al. (2018) suggested that the $D V L 2$ variant could be responsible for the "bulldog type" morphology and contribute to a Robinowlike syndrome in dogs.

The DVL2 variant segregates in a recessive manner with caudal vertebral malformations and has incomplete and variable penetrance for thoracic vertebral malformations (Mansour et al. 2018). However, since the allele is entirely or nearly fixed in English Bulldogs, French Bulldogs and Boston Terriers (Mansour et al. 2018), it has been unclear what the variant's effects are on other morphological features and whether heterozygosity causes any phenotype. Our study 
investigated the deletion allele distribution in these three breeds, confirming that the variant is fixed, and identified breeds with allele variation, which allowed us to dissect the effects of the allele on canine morphology.

\section{Materials and methods}

\section{Study cohort and DNA extraction}

EDTA blood samples were collected from a total of 1954 privately owned dogs: 165 Boston Terriers, 297 French Bulldogs, 211 English Bulldogs, 4 Olde English Bulldogges, 11 American Bulldogs, 285 American Staffordshire Terriers (AST), 714 Staffordshire Bull Terriers (SBT), 73 Dogues de Bordeaux, 16 Bull Terriers, 13 Miniature Bull Terriers, 40 Lhasa Apsos, 20 Shih Tzus, 46 Tibetan Spaniels, 10 Pekingese, 47 King Charles Spaniels and 2 mixed breed dogs (French Bulldog $\times$ German Shepherd Dog). Genomic DNA was extracted with a semi-automated Chemagic 360 extraction robot (PerkinElmer Chemagen Technologie $\mathrm{GmbH}$, Germany). DNA concentration was measured with Nanodrop ND-1000 UV/Vis Spectrophotometer (Nanodrop technologies, Wilmington, Delaware, USA) or DeNovix DS-11 Spectrophotometer (DeNovix Inc., Wilmington, Delaware, USA). The samples were stored at $-20^{\circ} \mathrm{C}$. Sample collection was approved by the Animal Ethics Committee of the State Provincial Office of Southern Finland (ESAVI/343/04.10.07/2016 and ESAVI/25696/2020).

\section{Variant screening}

We genotyped the DVL2 variant in the 1954 dogs with standard PCR and Sanger sequencing using the primers designed by Mansour et al. (2018): 5'-CGGCTAGCTGTCAGTTCT GG-3' (forward) and 5'-CAGTGAGTCTGAGCCCTCCA-3' (reverse). The amplified PCR products were sequenced with a capillary sequencer (Applied Biosystems ABI3730XL DNA Analyzer) at the Institute for Molecular Medicine Finland (FIMM) core facility. The sequences were analyzed using the Sequencher (version 5.3, GeneCodes, USA) and UGENE software (Okonechnikov et al. 2012).

\section{Prospective CT imaging}

We recruited nineteen ASTs (11 males and eight females) with known $D V L 2$ genotypes (eight wild types, eight heterozygotes, and three homozygotes) to a full-body CT examination at the University of Helsinki Veterinary Teaching Hospital. A physical examination was performed, body weight and age were recorded, and height at the withers was measured using measuring tape with bubble level.
Each dog was mildly sedated for the CT scan with 0.002-0.006 mg/kg dexmedetomidine (Dexdomitor; Orion, Finland) and $0.08-0.2 \mathrm{mg} / \mathrm{kg}$ butorphanol (Butordol; Intervet International B.V., Boxmeer, Netherlands) administered intramuscularly (IM) as a single injection. According to two dog owners, one dog had a mild aortic stenosis and another had an innocent murmur, both diagnosed by echocardiography. In these two cases, a veterinary anesthesiologist was consulted prior to sedation.

A non-contrast full-body helical CT imaging was performed in dorsal recumbency with GE LightSpeed VCT 64 (GE Healthcare, Fairfield, Connecticut). The parameters used were $120 \mathrm{kV}$ tube voltage, maximum current $750 \mathrm{mAs}$, noise index 10 , interval $0,625 \mathrm{~mm}, 0,625 \mathrm{~mm}$ slice thickness in both bone and soft tissue algorithms (bone window: center 500, width 1500 Hounsfield units; soft tissue window: center 40, width 400 Hounsfield units), using a $512 \times 512$ matrix. The acquisition extended from the tip of the nose to the tip of the tail.

In addition to the nineteen ASTs, we also recruited one wild-type SBT to a CT examination due to a short and kinked tail. CT imaging, physical examination and sedation were performed as described above.

\section{Radiographs}

Spinal radiographs of twenty-three ASTs (12 males, 11 females) with known $D V L 2$ genotype (21 wild types, 1 heterozygote, 1 homozygote) were retrieved from the Finnish Kennel Club's image database with the owner's informed consent and evaluated and measured retrospectively for this study. The set consisted of laterolateral images of cervical, thoracic and lumbar spine with the sacrum, and a ventrodorsal image of the pelvis. One dog also had laterolateral and ventrodorsal images of the tail. Age, gender, and body weight were also recorded.

As a pilot study, spinal and tail radiographs of three mixed breed dogs (French Bulldog $\times$ German Shepherd Dog) from the same litter were taken at the University of Helsinki Veterinary Teaching Hospital. The physical examination and sedation were performed as described above.

\section{Assessment and measurements of the images}

All images were interpreted by a clinical instructor with ten years of experience in radiology (VR), unaware of the dogs' DVL2 genotypes. CT image analysis was done using Horos software (v2.0.0 RC3 open-source PACS workstation DICOM viewer). Radiographs were assessed using Clear Canvas (Synaptive Medical, Toronto, Canada, version 7.0) PACS workstation DICOM viewer.

From the CT images, hemivertebrae were classified and all other vertebral malformations recorded as previously 
described (Gutierrez-Quintana et al. 2014). The presence of spinal degenerative changes and spondylosis deformans, calcified discs and any other abnormalities were also recorded. The vertebral segment was recorded for each vertebral malformation and for each patient. Transitional vertebrae were not counted in the number of caudal vertebral malformations. All thoracic, lumbar and sacral vertebrae were measured from multiplanar sagittal reformatted images. The maximum length of the dorsal and ventral halves of the vertebral body were recorded. The maximum height of the vertebral body was measured in the cranio-caudal midpoint. The length of the bony tail was measured, and the number of caudal vertebrae was counted.

The length of the radial axis was measured bilaterally from dorsal multiplanar reformatted images. Joint orientation lines were drawn along the proximolateral aspect and the proximomedial aspect of the radial head and along the distolateral aspect and the distomedial aspect of the distal radius (Fox et al. 2006). The length of the radius was measured between those lines.

Cephalometric measurements included facial length and width, cranial length and width, mandibular length, skull length and width and skull base length (Evans and de Lahunta 2013). Distance between the eyes was measured as the distance between the right and left frontolacrimal suture, nasal bone length was measured from the nasion to the rostral end of os nasale midline and hard palate length from caudal to the rostral end of the hard palate. Soft palate length and thickness were measured on sagittal view as previously published (Heidenreich et al. 2016). Finally, the following indices were calculated: skull index $=$ skull width $\times 100 /$ skull length, cranial index $=$ cranial width $\times 100 /$ cranial length, and facial index $=$ skull width $\times 100 /$ facial length (Evans and de Lahunta 2013).

From the radiographs, vertebral malformations were classified, measured and recorded similarly to the CT images. The length of the bony tail was measured from the laterolateral image of the dog with radiographs of the tail.

\section{Statistical analyses}

The association of the DVL2 genotype with body measurements was examined with linear models and generalized linear models. The data consisted of the same 19 ASTs that underwent $\mathrm{CT}$ examination. We examined the association of the dog's genotype with 11 measurements, ratios and indices: body weight, height at the withers, radius length, soft palate length, soft palate height, hard palate length, skull base length, ratio of hard palate length to skull base length, facial index, cranial index and skull index. These variables were explained with the dog's $D V L 2$ genotype (wild type/ heterozygote/homozygote) and sex. Dog's body weight was also included as an explanatory variable if this improved the model fit, as evaluated by a decrease of at least 2 units of the Akaike Information Criterion (AIC). This resulted in the inclusion of body weight only in the soft palate height model. Length of the radius of both the right and left front leg was measured and thus, we built a mixed model with genotype and sex as fixed variables and dog identity as a random variable.

Model fit was assessed thoroughly. First, different distributions and link functions were compared by fitting the model and visually examining the residuals with packages boot and rcompanion (Davison and Hinkley 1997; Canty and Ripley 2019; Mangiafico 2019). Based on visual examination, linear models were used in analyses of body weight, height at the withers, radius length (linear mixed model), soft palate length, hard palate length, skull base length, cranial index and skull index. For ratio of hard palate length to skull base length and facial index, we built generalized linear models with gamma distribution and log link function. For soft palate height, inverse gaussian distribution with a log link function provided the best fit. Second, after choosing the distribution and link function, outliers were examined and plotted with packages broom, dplyr and ggplot2 (Wickham 2016; Robinson and Hayes 2019; Wickham et al. 2019). Third, multicollinearity was evaluated with variance inflation factor (VIF) using the package car (Fox and Weisberg 2019). Finally, the linearity of the continuous explanatory variable, body weight, was assessed by fitting a generalized additive model with the package gam (Hastie 2019).

To estimate the overall effect of variables, an analysis of variance (ANOVA) was run with the package car (Fox and Weisberg 2019). To obtain mean estimates for categorical variables and pairwise comparisons between levels of categorical variables, we calculated the estimated marginal means with the package emmeans (Lenth 2019). The estimates for the continuous variable were obtained with the package effects (Fox 2003; Fox and Weisberg 2019). Due to a high number of analyses and pairwise comparisons, all $p$-values were controlled for false discovery rate (FDR). The significance cut-off value was set to $p<0.05$. All statistical analyses were conducted with $\mathrm{R}$ version 3.6.2 ( $\mathrm{R}$ Core Team 2019).

\section{Results}

\section{Prevalence of the DVL2 variant}

We genotyped the $D V L 2$ variant in English Bulldogs, French Bulldogs and Boston Terriers to investigate the prevalence of the deletion allele in a large cohort. To identify breeds with $D V L 2$ allele variation and enable the investigation of the phenotype-genotype correlation, we also screened other breeds in which the allele segregates (Mansour et al. 2018) 
and closely related breeds. Furthermore, King Charles Spaniels were included due to a high prevalence of significant caudal vertebral anomalies (Hytönen et al. 2009). In total, the cohort included 1954 dogs (Table 1).

The DVL2 variant was completely fixed in Boston Terriers, French Bulldogs and English Bulldogs. Deletion homozygotes and heterozygotes were additionally discovered in ASTs and SBTs. Furthermore, heterozygotes were found in Dogues de Bordeaux, Olde English Bulldogges and American Bulldogs. Overall, the carrier frequency in these five breed cohorts ranged from $18.2 \%$ to $50.0 \%$; however, sample sizes were highly variable (from 4 to 708 ). Finally, the variant was absent in the seven other breeds.

\section{Clinical findings}

To dissect the effect of the DVL2 deletion on canine morphology, we carried out full-body CT examinations (Online material 1, Suppl. Tables 1-5) and retrospectively assessed radiographic images in a cohort of dogs with known genotypes (Online material 2, Suppl. tables 6-8). We chose to perform the examinations on ASTs because, among breeds with allele variation, the number of homozygous dogs was the highest. Nineteen dogs (11 males, eight females) participated in the CT examinations. The mean age of the cohort was 5.7 years (min: 2.7, max: 10.6, SD: 2.8), and the average weight was $29.2 \mathrm{~kg}$ in males (min: 23.0, max: 36.5, SD: 3.3 ) and $25.2 \mathrm{~kg}$ in females (min: 21.4, max: 29.2, SD: 2.5).
Among the 19 dogs, 3 were homozygous, 8 were heterozygous and 8 were wild-type for the $D V L 2$ variant.

The tail could be completely evaluated in all of the CT imaged dogs and in the radiographs of one DVL2 homozygote $(N=20)$. In the $D V L 2$ homozygous dogs $(N=4)$, mean tail length was $26 \mathrm{~cm}$, which was below the mean of heterozygous ( $33 \mathrm{~cm}, N=8)$ and wild-type $(34 \mathrm{~cm}, N=8)$ dogs. The total number of caudal vertebrae ranged from 19 to 21 in heterozygous and wild-type dogs and from 20 to 21 in homozygous dogs, all of which had abnormal vertebrae in the tail (Fig. 1). In the DVL2 homozygotes, the number of malformed caudal vertebrae per dog was $1,8,10$ and 14 , respectively. One or several of the following caudal vertebral malformations were detected: block vertebra, dorso-lateral hemivertebra, lateral hemivertebra, butterfly vertebra, ventral wedge shape, unclassified congenital malformation and abnormally short vertebra. Additionally, one homozygote had vertebral malformations in the sacrum, namely ventral wedge shape vertebra and an unclassified congenital malformation. No other vertebral malformations were detected in these four homozygous dogs. Finally, the owners of two homozygous dogs unavailable for a clinical examination (one AST and one SBT) reported that their dogs' tails were kinked.

In addition to the $D V L 2$ homozygotes, several heterozygous and wild-type dogs with tail anomalies were discovered in CT examinations and in the rest of the screening cohort (Fig. 2, Table 2). First, three out of the eight CT examined $D V L 2$ heterozygotes exhibited caudal vertebral

Table 1 Frequency of the $D V L 2$ deletion allele in the studied breeds

\begin{tabular}{|c|c|c|c|c|c|c|}
\hline Breed & $\mathrm{wt} / \mathrm{wt}(N)$ & wt/del $(N)$ & $\operatorname{del} / \operatorname{del}(N)$ & Total $(N)$ & wt/del (\%) & del/del (\%) \\
\hline Boston Terrier* & 0 & 0 & 165 & 165 & 0 & 100 \\
\hline French Bulldog* & 0 & 0 & 211 & 211 & 0 & 100 \\
\hline English Bulldog* & 0 & 0 & 297 & 297 & 0 & 100 \\
\hline American Staffordshire Terrier (AST) & 201 & 79 & 5 & 285 & 27.7 & 1.8 \\
\hline Staffordshire Bull Terrier (SBT) * & 578 & 135 & 1 & 714 & 18.9 & 0.1 \\
\hline Dogue de Bordeaux & 46 & 27 & 0 & 73 & 37.0 & 0 \\
\hline Olde English Bulldogge & 2 & 2 & 0 & 4 & 50.0 & 0 \\
\hline American Bulldog & 9 & 2 & 0 & 11 & 18.2 & 0 \\
\hline Bull Terrier & 16 & 0 & 0 & 16 & 0 & 0 \\
\hline Miniature Bull Terrier & 13 & 0 & 0 & 13 & 0 & 0 \\
\hline Lhasa Apso & 40 & 0 & 0 & 40 & 0 & 0 \\
\hline Shih Tzu* & 20 & 0 & 0 & 20 & 0 & 0 \\
\hline Tibetan Spaniel & 46 & 0 & 0 & 46 & 0 & 0 \\
\hline Pekingese & 10 & 0 & 0 & 10 & 0 & 0 \\
\hline King Charles Spaniel & 47 & 0 & 0 & 47 & 0 & 0 \\
\hline $\begin{array}{l}\text { Mixed breed (French Bulldog/German } \\
\text { Shepherd Dog) }\end{array}$ & 0 & 2 & 0 & 2 & & \\
\hline Total & 1028 & 247 & 679 & 1954 & & \\
\hline
\end{tabular}

Breeds that have previously been found to carry the allele (Mansour et al. 2018) are denoted with an asterisk $(*)$ 
Fig. 1 Volume rendering technique (3D) computed tomography images of tails of four American Staffordshire Terriers. a-c are $D V L 2$ homozygous dogs with varying number and types of vertebral malformations. The dog in image A with most malformations had also abnormal sacral vertebrae. d Normal tail of a DVL2 wild-type dog

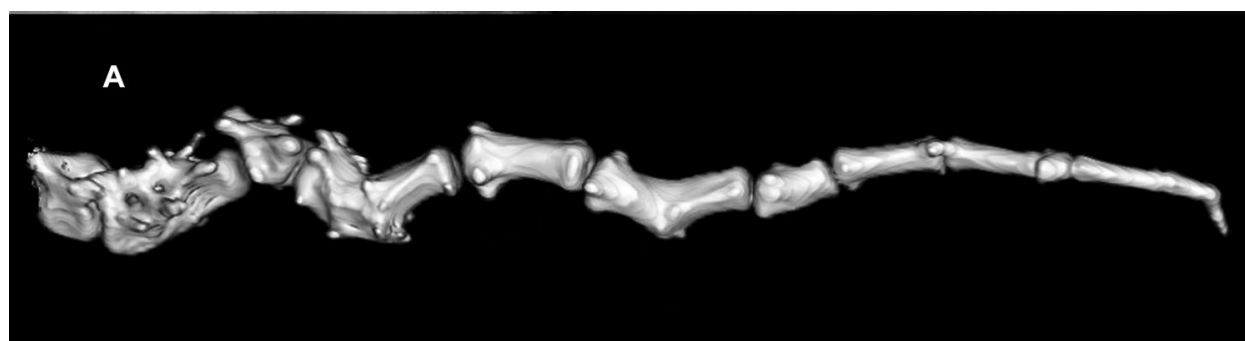

B

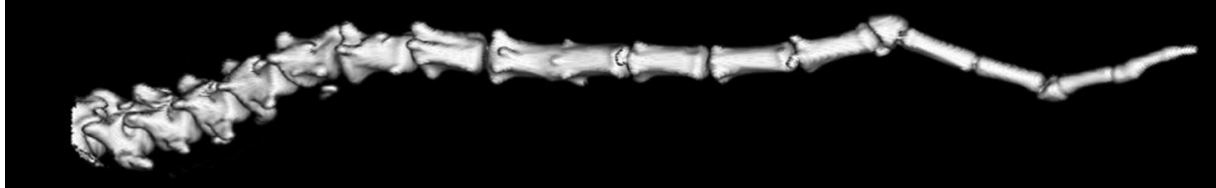

C

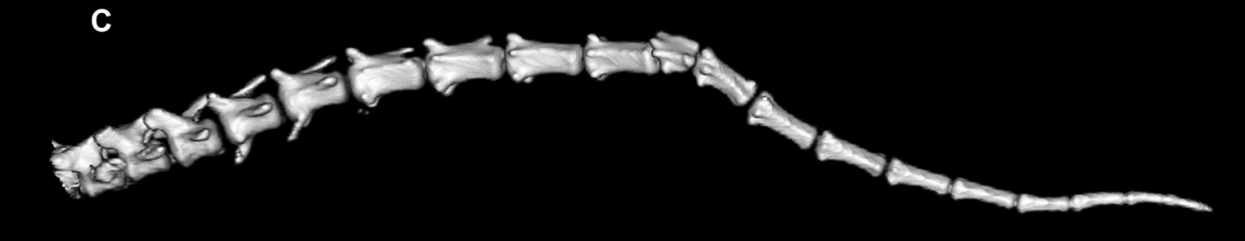

D

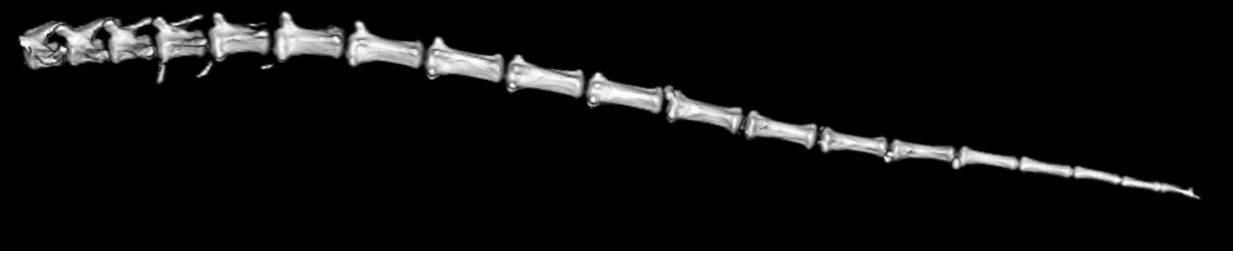

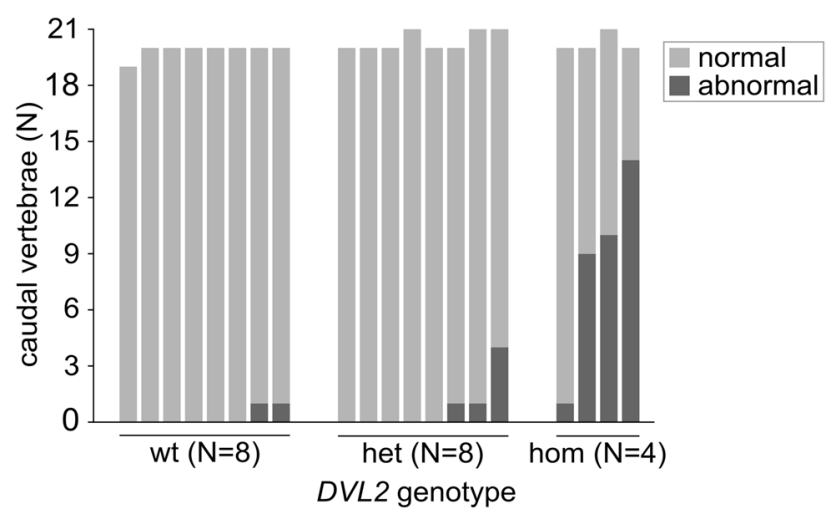

Fig. 2 The number of normal and abnormal caudal vertebrae in by $D V L 2$ genotype in one radiographed and nineteen CT examined American Staffordshire Terriers

malformations: one had four malformations (ventral wedge shape and dorsal hemivertebra and two unclassified congenital malformations) and two dogs had one malformation (unclassified congenital malformation). Second, two out of the eight CT examined wild-type ASTs had one caudal vertebral malformation (dorso-lateral hemivertebra and unclassified congenital malformation). Additionally, one
CT examined wild-type SBT had a short tail consisting of only 11 caudal vertebrae, which is less than the average of 20 caudal vertebrae typically seen in dogs with normal tails such as our wild-type ASTs. The penultimate vertebra had an unclassified congenital malformation, and the last vertebra was small, triangular and pointing dorsally. The other caudal vertebrae were normal. Thoracic, lumbar or sacral vertebral malformations were not detected in any DVL2 heterozygotes or wild types.

In total, 12 heterozygous and 13 wild-type dogs in the screening cohorts had tail anomalies either observed in CT studies or according to the owner's report (Table 2). Notably, accurate health information was not available for the entire screening cohorts, so the frequency of tail anomalies is not representative of the entire breeds and possibly an underestimation. Finally, all three French Bulldog $\times$ German Shepherd crosses had normal spines and normal-length tails without any vertebral malformations.

Shape of the cervical, thoracic and lumbar vertebrae and the ratio of the height to the length of the thoracic and lumbar vertebrae were normal in all dog groups. Spondylosis and features of transitional vertebra were found among all $D V L 2$ genotypes. 
Table 2 The number of tail anomalies in heterozygous and wild-type dogs observed in CT examinations or reported by the owner

\begin{tabular}{lllll}
\hline Breed & Source & \multicolumn{2}{l}{$\begin{array}{l}\text { Dogs with tail anoma- } \\
\text { lies }(N)\end{array}$} & \begin{tabular}{l} 
Screening \\
cohort \\
\cline { 3 - 4 }
\end{tabular} \\
\cline { 3 - 4 } & & het & wt & \\
\hline American Staffordshire Terrier (AST) & CT examination & 3 & 2 & 280 \\
& Owner report & 1 & 0 & \\
Staffordshire Bull Terrier (SBT) & CT examination & NA & 1 & 713 \\
& Owner report & 5 & 8 & \\
Dogue de Bordeaux & Owner report & 2 & 2 & 73 \\
Olde English Bulldogge & Owner report & 1 & 0 & 4 \\
American Bulldog & Owner report & 0 & 0 & 11 \\
& Total & 12 & 13 & 1081 \\
\hline
\end{tabular}

Het heterozygous, wt wild-type
Finally, two of the three DVL2 homozygotes (one male and one female) in the CT study had asymmetrical frontal sinuses and a mild heart murmur in clinical examination. One dog had previously been diagnosed with aortic stenosis in echocardiography and the other had had echocardiographic measurements within the normal range, but a follow-up examination by a cardiologist had been recommended. Additionally, one homozygous AST unavailable for CT examination had a mild, asymptomatic heart murmur according to the owner's report.

\section{Association of the DVL2 genotype with body measurements}

Of the 11 body measurements, five were associated with $D V L 2$ genotype: hard palate length, skull base length, the ratio of hard palate length to skull base length, facial index (Fig. 3) and soft palate height. Additionally, the sex of the dog was associated with body weight, height at withers, radius length and hard palate length. Finally, soft palate height was associated with the bodyweight of the dog, with a thicker soft palate correlating with higher body weight.

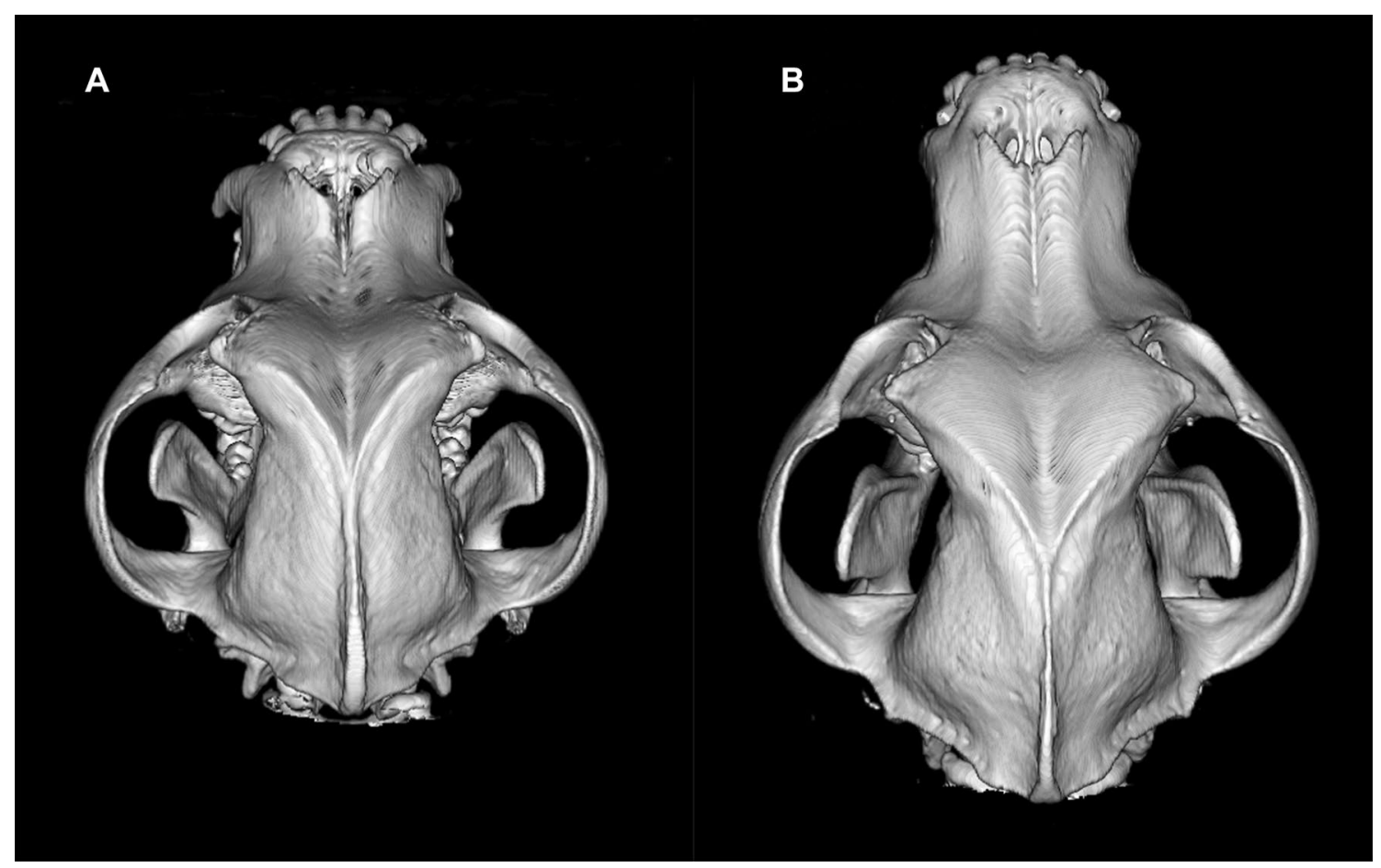

Fig. 3 Volume rendering technique (3D) computed tomography images of skulls of two American Staffordshire Terriers. a DVL2 homozygote. b $D V L 2$ wild type. Differences in the skull and facial lengths between genotypes are evident 
The DVL2 genotype was significantly associated with hard palate length: homozygotes had a shorter hard palate than heterozygotes $(Z=-5.9, \mathrm{df}=1, P<0.0001)$ and wildtype dogs $(Z=-8.8, \mathrm{df}=1, P<0.0001)$ and heterozygotes had a shorter hard palate than wild types $(Z=-4.0, \mathrm{df}=1$, $P=0.007$; Fig. 4a). However, the length of the soft palate did not differ between genotypes $(F=0.28, \mathrm{df}=2, P=0.80$; Fig. 4f; see pairwise comparisons in Suppl. Table 17). Skull

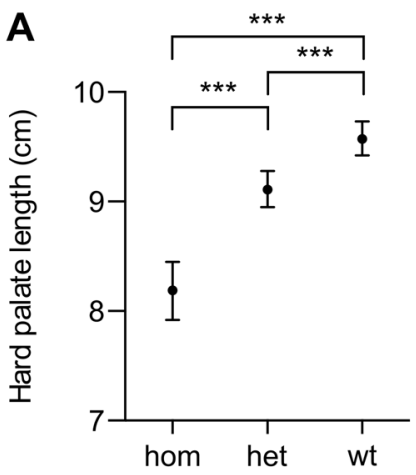

D

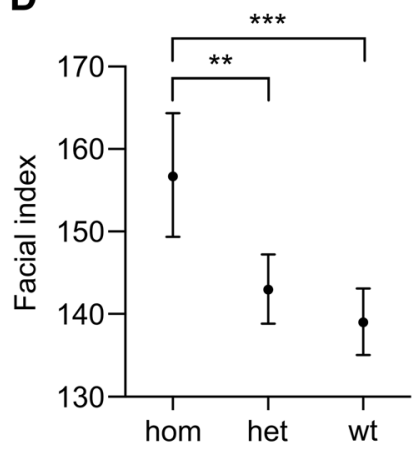

B

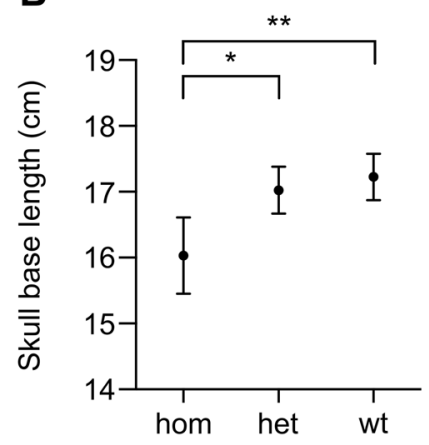

E

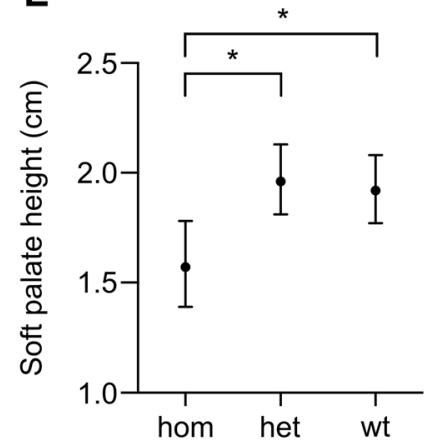

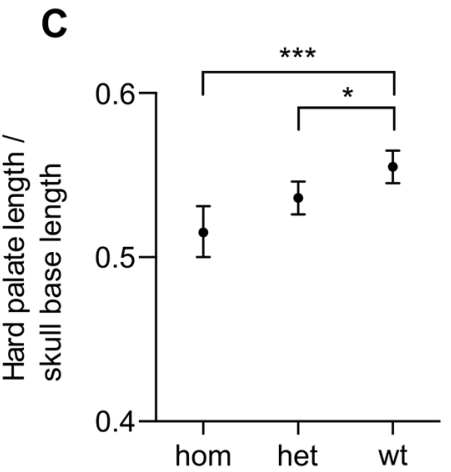

$\mathbf{F}$

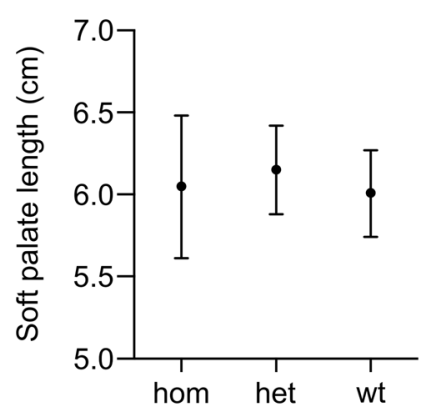

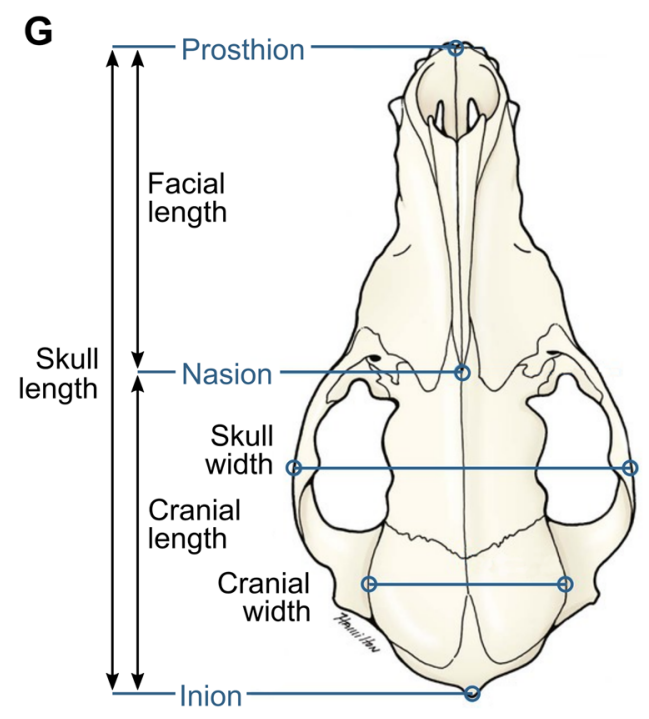

Fig. 4 Association of DVL2 genotypes with body measurements traits, and schematic representation of a dog skull. Asterisks *, ** and $* * *$ indicate the significance of $p<0.05, p<0.01$ and $p<0.001$, respectively. a The length of the hard palate differed between all genotypes. b The length of the skull base differed between homozygotes and other genotypes. c The ratio of hard palate length to skull base length was smaller in non-wild-type than wild-type dogs. d Facial index was higher in homozygotes than other genotypes. e The height

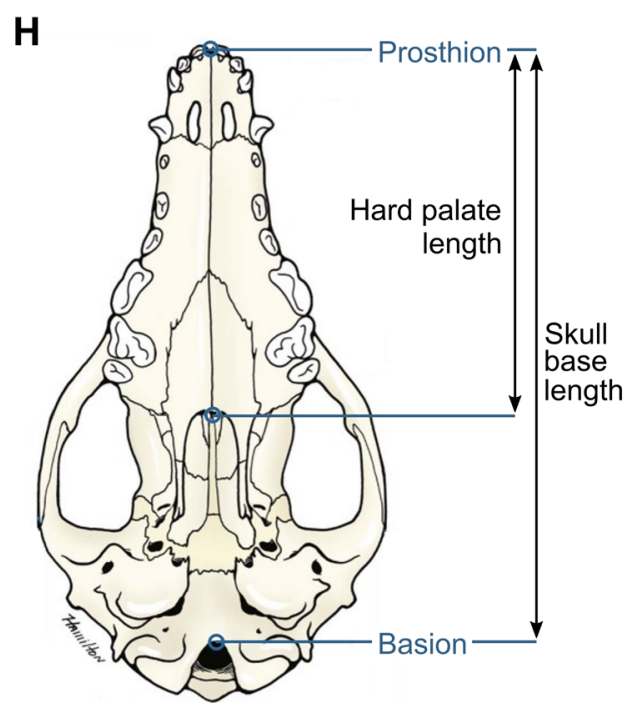

of the soft palate differed between homozygotes and other genotypes. f Genotype was not associated with the length of the soft palate. $\mathbf{g}$ Dorsal view of a dog skull with craniometrics points. $\mathbf{h}$ Ventral view of a dog skull with craniometric points Images $\mathbf{g}$ and $\mathbf{h}$ are adapted from Miller's Anatomy of the Dog (4th edition) by Evans $\mathrm{H}$ and de Lahunta A, p. 86-87, 2012, Elsevier Health Sciences. Copyright Elsevier (2013). Reprinted with permission 
base was shorter in homozygotes than in heterozygotes $(Z=-2.9, \mathrm{df}=1, P=0.014)$ and wild-types $(Z=-3.4$, $\mathrm{df}=1, P=0.003$; Fig. 4b). The ratio of hard palate length to skull base length was also lower in homozygotes $(Z=-4.2$, $\mathrm{df}=1, P=0.0003)$ and heterozygotes $(Z=-2.6, \mathrm{df}=1$, $P=0.028$ ) than in wild types (Fig. 4c). Furthermore, the facial index was higher in homozygotes than in heterozygotes $(Z=3.2, \mathrm{df}=1, P=0.006)$ and wild types $(Z=4.2$, $\mathrm{df}=1, P=0.0003$; Fig. 4d). Finally, soft palate height was smaller in homozygotes than in heterozygotes $(Z=-3.0$, $\mathrm{df}=1, P=0.010)$ and wild types $(Z=-2.6, \mathrm{df}=1$, $P=0.028$; Fig. 4e). ANOVA tables are found in Online material 3, Supplementary tables 9-11 and all pairwise contrasts between genotypes in Online material 3, Supplementary tables 12-22.

\section{Discussion}

We screened the Robinow-like syndrome associated $D V L 2$ variant in 15 breeds, consisting of mainly Bulldog and Pit Bull type dogs as well as Tibetan dogs, to better understand the distribution of the deletion allele in canine populations. Like Mansour et al. (2018), we found that the variant is fixed in French Bulldogs and English Bulldogs. Likewise, all Boston Terriers in our study were homozygous, although a 94\% homozygote frequency was observed by Mansour et al. (2018). Additionally, we found the allele in American Staffordshire Terriers, Staffordshire Bull Terriers, Dogues de Bordeaux, American Bulldogs, and Olde English Bulldogges. In ASTs and SBTs, some homozygous individuals were found, which enabled the investigation of the phenotypegenotype correlation to dissect the specific effects of the DVL2 variant.

To elucidate the quantitative and qualitative effects of the DVL2 deletion allele on canine morphology, we recruited 19 ASTs to a full-body CT examination and retrospectively assessed radiographic images of $23 \mathrm{dogs}$. We found that all examined homozygotes had variable caudal vertebral malformations, which is consistent with previous results; however, the total number of caudal vertebrae was not reduced and the tail was not as completely malformed and fused as in English Bulldogs, French Bulldogs and Boston Terriers (Mansour et al. 2018). Additionally, one homozygous dog had sacral vertebral malformations, but lumbar or thoracic abnormalities were not detected in any dogs, including the German Shepherd Dog $\times$ French Bulldog crosses, and the shape of the vertebrae were similar in all dogs. This is in sharp contrast to English Bulldogs, French Bulldogs and Boston Terriers, in which previous reports have found a high prevalence of vertebral malformations (Moissonnier et al. 2011; Guevar et al. 2014; Gutierrez-Quintana et al. 2014; Ryan et al. 2017). Our results indicate that either the deletion does not result in thoracic malformations or their penetrance is more variable than the previously estimated $45-100 \%$ (Mansour et al. 2018). The incomplete penetrance might also reflect the complex role of $D V L 2$ in skeletal development, as vertebral malformations were similarly not fully penetrant in Dvl2 $2^{-/}$mice, where $90 \%$ of embryos exhibited abnormal vertebral bodies and $25 \%$ of surviving offspring had kinked tails (Hamblet et al. 2002). Conversely, caudal vertebral malformations were also present in heterozygous and wild-type dogs, and the allele was not found in King Charles Spaniels, which together indicate that besides the DVL2 allele, there are additional, still unknown variants that cause tail kinks in dogs. This is further supported by studies in mice, where kinked tail (MP:0000585) is associated with more than 370 genotypes in different strains (Smith and Eppig 2009; Bult et al. 2019).

In addition to the caudal morphological anomalies, we found that the $D V L 2$ deletion significantly affects several cephalometric measurements. Specifically, a shorter hard palate and skull base length, a smaller ratio of hard palate length to skull base length, and a higher facial index were associated with one or two DVL2 deletion alleles, which together confirm that the variant results in a more brachycephalic phenotype (Fig. 1). In contrast, the length of the soft palate was not associated with genotype, indicating that it is of similar length in all genotypes despite the reduced facial skeleton length, which results in a mismatch in proportions of the facial skeleton and soft tissues of the oral cavity, i.e., an elongated soft palate. Importantly, elongated soft palate, together with stenotic nares, is a primary abnormality in brachycephalic obstructive airway syndrome (BOAS) (Stockard 1941; Harvey 1989). Shortening of the skeletal muzzle results in a cramming effect, with the "excess" soft tissues interfering with airflow and partially blocking the nasopharynx and the larynx during respiration (Harvey 1989). Stenotic nares further increase airflow resistance and impair thermoregulation (Knecht 1979; Oechtering et al. 2010). As a result, affected individuals have clinical signs such as respiratory distress, dyspnea, and heat and exercise intolerance, and surgery of the soft palate and nares as well as secondary changes, such as laryngeal collapse, is often needed to reduce airway obstruction and improve quality of life (Packer and Tivers 2015).

Our results strongly indicate brachycephaly and elongated soft palate in dogs with the DVL2 deletion allele and suggest that additional risk for BOAS may be conferred by the variant. However, our cohort size was very small, with only three homozygotes, and the allele acted with variable mechanisms, including incompletely dominant (hard palate length), fully dominant (ratio of hard palate length to skull base length) or recessive (skull base length, facial index and soft palate height), depending on the measurement. Thus, our results need to be confirmed in a larger cohort before a 
definitive mode of inheritance can be determined for each trait.

In addition to altered facial proportions, mesomelic limb shortening is a typical feature in human Robinow patients (White et al. 2018). In contrast, while our data did show a slight trend between DVL2 genotype and a shorter radius as well as the lower height at withers, the results were not statistically significant (Online Material 3, Suppl. tables 13-14). These body proportions will also need to be studied in a larger cohort to confirm whether mesomelic dwarfism is a part of the phenotype in DVL2 homozygous dogs.

As an additional discovery, we found that two out of three homozygous dogs in the CT study had a mild heart murmur, and one of them had a previously confirmed diagnosis of aortic stenosis. During organogenesis, aortic and pulmonary valves arise from endocardial cushions with the involvement of cardiac neural crest cells, and these cell populations are also involved in the septation of the common outflow tract into the aorta and pulmonary trunk (Waldo et al. 1998; Nakajima et al. 2000; Jiang et al. 2000; Délot 2003; Brown and Baldwin 2006; Hutson and Kirby 2007). Notably, Dvl2 ${ }^{-/-}$mice exhibit various outflow tract malformations, including double outlet right ventricle, transposition of the great arteries, and persistent truncus arteriosus, due to a signaling defect in the cardiac neural crest (Hamblet et al. 2002). Based on this common developmental origin of the outflow tract and semilunar valves, it can be hypothesized that the DVL2 deletion may be involved in cardiovascular anomalies in homozygous dogs. However, this suggestion is speculative and warrants further research, especially since pulmonary stenosis and aortic stenosis are among the most common congenital heart defects in English Bulldogs, French Bulldogs and ASTs (Brambilla et al. 2020).

Screw tail is a breed characteristic in Boston Terriers, French Bulldogs and English Bulldogs, and they are fixed for the DVL2 deletion. In contrast, caudal vertebral anomalies are undesired and selected against in ASTs, SBTs and Dogues de Bordeaux, which is paralleled by the lower allele frequency (18.9-37.0\%) in these breeds. Notably, the genotypes observed are in Hardy-Weinberg equilibrium in ASTs but not in SBTs despite a large number of dogs. Based on the frequency of the DVL2 allele in SBTs, we would have expected to find more deletion homozygotes (observed $=1$, expected $=6.6, P<0.015)$. Whether this reflects a sampling bias or some other phenomenon, e.g. removal of homozygous dogs from the population, is currently unknown to us.

Altogether, the quantitative and qualitative morphological changes confirm that the DVL2 deletion results in a syndromic phenotype resembling Robinow syndrome in humans, supporting the previous hypothesis of the canine Robinow-like syndrome. Still, the features on the spectrum of human Robinow syndrome are not influenced solely by DVL2 in dogs: as an example, the brachycephalic, short-limbed and vertebral phenotypes are more extreme in English Bulldogs, French Bulldogs and Boston Terriers due to selective breeding. In general, many morphological traits in dogs are genetically complex and affected by multiple variants, with their frequencies influenced by differential selective pressures in different breeds. Examples of such variants include the brachycephaly associated SMOC2 and BMP3 variants and chondrodysplasia-associated FGF4 retrogenes on chromosomes 12 and 18 (Parker et al. 2009; Schoenebeck et al. 2012; Marchant et al. 2017; Brown et al. 2017), and there are likely more, yet undiscovered variants. Additionally, it is notable that in zebrafish, zygotic dv12 mutants are relatively normal compared to wild-type individuals, whereas maternal-zygotic mutants exhibit severe craniofacial defects resembling a "bulldog facial phenotype" (Xing et al. 2018). To our knowledge, the possible maternal effect of DVL2 in mammals has not been studied. Thus, it can be hypothesized that Robinowlike syndrome could also be more extensive in dogs with deletion homozygous dams, which is always the case in breeds where the allele is fixed. However, further research is needed to test whether our hypothesis is correct.

In summary, our study strengthens the role of the DVL2 variant in the brachycephalic phenotype and caudal vertebral anomalies, and suggests that other conditions may be linked to canine Robinow-like syndrome, including BOAS and congenital heart defects. Kinked tail is an undesired trait in non-screw tail breeds and our study shows that screening of the DVL2 variant could help to redesign breeding plans to reduce unwanted $D V L 2$-related anomalies and improve canine welfare.

Supplementary Information The online version contains supplementary material available at https://doi.org/10.1007/s00439-021-02261-8.

Acknowledgements We thank Rosanna Lehmusto, Sini Karjalainen, and Ileana Quintero for their technical assistance. The Institute for Molecular Medicine Finland (FIMM) is acknowledged for the Sanger sequencing, and CSC-IT Center for Science for computational resources. Finally, we are grateful to the dog breeders and owners for participating in this study.

Author contributions Conceptualization: HL and MKH; Study design: MKH, HL, JEN, VR, AKL, DB; Investigation and formal analysis: JEN, VR, MS; Resources: HL, VR; Writing—original draft: JEN, VR, MS; Writing_editing and review: all; Supervision: HL, MKH; Funding acquisition: HL, MKH.

Funding Open access funding provided by University of Helsinki including Helsinki University Central Hospital. This study was partially supported by the Dog Health Research Fund, Integrative Life Science Doctoral Program, Jane and Aatos Erkko Foundation, the Academy of Finland, and Helsinki Institute of Life Science.

Data availability All data generated or analysed during this study are included in this published article and its supplementary information files. 


\section{Compliance with ethical standards}

Conflict of interest No conflicts of interest.

Ethical approval Sample collection was ethically approved by the Animal Ethics Committee of the State Provincial Office of Southern Finland (ESAVI/343/04.10.07/2016 and ESAVI/25696/2020).

Consent to participate Written consent was given by the dogs' owners that donated samples from their dogs or participated in clinical examinations.

Open Access This article is licensed under a Creative Commons Attribution 4.0 International License, which permits use, sharing, adaptation, distribution and reproduction in any medium or format, as long as you give appropriate credit to the original author(s) and the source, provide a link to the Creative Commons licence, and indicate if changes were made. The images or other third party material in this article are included in the article's Creative Commons licence, unless indicated otherwise in a credit line to the material. If material is not included in the article's Creative Commons licence and your intended use is not permitted by statutory regulation or exceeds the permitted use, you will need to obtain permission directly from the copyright holder. To view a copy of this licence, visit http://creativecommons.org/licenses/by/4.0/.

\section{References}

Brambilla PG, Polli M, Pradelli D, Papa M, Rizzi R, Bagardi M, Bussadori C (2020) Epidemiological study of congenital heart diseases in dogs: Prevalence, popularity, and volatility throughout twenty years of clinical practice. PLoS ONE 15:e0230160. https://doi. org/10.1371/journal.pone.0230160

Brown CB, Baldwin HS (2006) Neural crest contribution to the cardiovascular system neural crest induction and differentiation. Springer US, Boston, pp 134-154

Brown EA, Dickinson PJ, Mansour T, Sturges BK, Aguilar M, Young AE, Korff C, Lind J, Ettinger CL, Varon S, Pollard R, Brown CT, Raudsepp T, Bannasch DL (2017) FGF4 retrogene on CFA12 is responsible for chondrodystrophy and intervertebral disc disease in dogs. PNAS 114:11476-11481

Bult CJ, Blake JA, Smith CL, Kadin JA, Richardson JE (2019) Mouse genome database (MGD) 2019. Nucleic Acids Res 47:D801D806. https://doi.org/10.1093/nar/gky1056

Canty A, Ripley BD (2019) boot: Bootstrap R (S-Plus) Functions. R package version $1.3-25$

Davison AC, Hinkley DV (1997) Bootstrap methods and their application. Cambridge University Press, New York

Délot EC (2003) Control of endocardial cushion and cardiac valve maturation by BMP signaling pathways. Mol Genet Metab 80:27-35. https://doi.org/10.1016/j.ymgme.2003.07.004

Evans H, de Lahunta A (2013) Miller's Anatomy of the Dog. Saunders, Philadelphia

Fox J (2003) Effect displays in R for generalised linear models. J Stat Softw 8:1-27. https://doi.org/10.18637/jss.v008.i15

Fox J, Weisberg S (2019) An R companion to applied regression. Sage, Los Angeles

Fox DB, Tomlinson JL, Cook JL, Breshears LM (2006) Principles of uniapical and biapical radial deformity correction using dome osteotomies and the center of rotation of angulation methodology in dogs. Vet Surg 35:67-77. https://doi.org/10.1111/j.1532950X.2005.00114.x
Guevar J, Penderis J, Faller K, Yeamans C, Stalin C, Gutierrez-Quintana $\mathrm{R}$ (2014) Computer-assisted radiographic calculation of spinal curvature in brachycephalic "Screw-Tailed" dog breeds with congenital thoracic vertebral malformations: reliability and clinical evaluation. PLoS ONE 9:e106957. https://doi.org/10.1371/ journal.pone.0106957

Gutierrez-Quintana R, Guevar J, Stalin C, Faller K, Yeamans C, Penderis J (2014) A proposed radiographic classification scheme for congenital thoracic vertebral malformations in brachycephalic "screw-tailed" dog breeds. Vet Radiol Ultrasound 55:585-591. https://doi.org/10.1111/vru.12172

Hamblet NS, Lijam N, Ruiz-Lozano P, Wang J, Yang Y, Luo Z, Mei L, Chien KR, Sussman DJ, Wynshaw-Boris A (2002) Dishevelled 2 is essential for cardiac outflow tract development, somite segmentation and neural tube closure. Development (Cambridge) 129:5827-5838. https://doi.org/10.1242/dev.00164

Harvey CE (1989) Inherited and congenital airway conditions. J Small Anim Pract 30:184-187. https://doi. org/10.1111/j.1748-5827.1989.tb01531.x

Hastie T (2019) gam: generalized additive models. R package version 1.16.1. https://CRAN.R-project.org/package $=$ gam

Heidenreich D, Gradner G, Kneissl S, Dupré G (2016) Nasopharyngeal dimensions from computed tomography of pugs and French bulldogs with brachycephalic airway syndrome. Vet Surg 45:83-90. https://doi.org/10.1111/vsu.12418

Hutson MR, Kirby ML (2007) Model systems for the study of heart development and disease: cardiac neural crest and conotruncal malformations. Semin Cell Dev Biol 18:101-110. https://doi. org/10.1016/j.semcdb.2006.12.004

Hytönen MK, Grall A, Hédan B, Dréano S, Seguin SJ, Delattre D, Thomas A, Galibert F, Paulin L, Lohi H, Sainio K, André C (2009) Ancestral T-box mutation is present in many, but not all, short-tailed dog breeds. J Hered 100:236-240. https://doi. org/10.1093/jhered/esn085

Jiang X, Rowitch DH, Soriano P, McMahon AP, Sucov HM (2000) Fate of the mammalian cardiac neural crest. Development 127:1607-1616

Knecht CD (1979) Upper airway obstruction in brachycephalic dogs. Compend Contin Educ Pract Vet 1:25-31

Lenth R (2019) emmeans: estimated marginal means, aka LeastSquares Means. R package version 1.4.3.01. https://CRAN.Rproject.org/package=emmeans

Mangiafico S (2019) Functions to support extension education program evaluation. R package version 2.3.7. https://CRAN.R-project.org/ package $=$ rcompanion

Mansour TA, Lucot K, Konopelski SE, Dickinson PJ, Sturges BK, Vernau KL, Choi S, Stern JA, Thomasy SM, Döring S, Verstraete FJM, Johnson EG, York D, Rebhun RB, Ho HH, Brown CT, Bannasch DL (2018) Whole genome variant association across 100 dogs identifies a frame shift mutation in DISHEVELLED 2 which contributes to Robinow-like syndrome in Bulldogs and related screw tail dog breeds. PLoS Genet 14:e1007850. https://doi. org/10.1371/journal.pgen.1007850

Marchant TW, Johnson EJ, McTeir L, Johnson CI, Gow A, Liuti T, Kuehn D, Svenson K, Bermingham ML, Drögemüller M, Nussbaumer M, Davey MG, Argyle DJ, Powell RM, Guilherme S, Lang J, Ter Haar G, Leeb T, Schwarz T, Mellanby RJ, Clements DN, Schoenebeck JJ (2017) Canine brachycephaly is associated with a retrotransposon-mediated missplicing of SMOC2. Curr Biol 27:1573-1584.e6. https://doi.org/10.1016/j.cub.2017.04.057

Moissonnier P, Gossot P, Scotti S (2011) Thoracic kyphosis associated with hemivertebra. Vet Surg 40:1029-1032. https://doi. org/10.1111/j.1532-950X.2011.00876.X

Nakajima Y, Yamagishi T, Hokari S, Nakamura H (2000) Mechanisms involved in valvuloseptal endocardial cushion formation in early cardiogenesis: roles of transforming growth factor (TGF)- $\beta$ and 
bone morphogenetic protein (BMP). Anat Rec 258:119-127. https ://doi.org/10.1002/(SICI)1097-0185(20000201)258:23.0.CO;2-U

Oechtering G, Schlüter C, Lippert J (2010) Brachycephaly in dog and cat: a "human induced" obstruction of the upper airways. Pneumologie 64:450-452. https://doi.org/10.1055/s-0030-1255513

Okonechnikov K, Golosova O, Fursov M (2012) Unipro UGENE: a unified bioinformatics toolkit. Bioinformatics 28:1166-1167. https://doi.org/10.1093/bioinformatics/bts091

Packer RM, Tivers MS (2015) Strategies for the management and prevention of conformation-related respiratory disorders in brachycephalic dogs. Veterinary Med (Auckland) 6:219-232. https://doi. org/10.2147/VMRR.S60475

Parker HG, VonHoldt BM, Quignon P, Margulies EH, Shao S, Mosher DS, Spady TC, Elkahloun A, Cargill M, Jones PG, Maslen CL, Acland GM, Sutter NB, Kuroki K, Bustamante CD, Wayne RK, Ostrander EA (2009) An expressed Fgf4 retrogene is associated with breed-defining chondrodysplasia in domestic dogs. Sci (Am Assoc Adv Sci) 325:995-998. https://doi.org/10.1126/scien ce. 1173275

R Core Team (2019) R: a language and environment for statistical computing. R Foundation for Statistical Computing, Vienna

Robinson D, Hayes A (2019) broom: convert statistical analysis objects into tidy tibbles. R package version 0.5.2. https://CRAN.R-proje ct.org/package $=$ broom

Ryan R, Gutierrez-Quintana R, ter Haar G, De Decker S (2017) Prevalence of thoracic vertebral malformations in French bulldogs, Pugs and English bulldogs with and without associated neurological deficits. Vet J 221:25-29. https://doi.org/10.1016/j. tvj1.2017.01.018

Schoenebeck JJ, Hutchinson SA, Byers A, Beale HC, Carrington B, Faden DL, Rimbault M, Decker B, Kidd JM, Sood R, Boyko AR, Fondon JW, Wayne RK, Bustamante CD, Ciruna B, Ostrander EA (2012) Variation of BMP3 contributes to dog breed skull diversity. PLoS Genet 8:e1002849. https://doi.org/10.1371/journ al.pgen.1002849

Smith CL, Eppig JT (2009) The mammalian phenotype ontology: enabling robust annotation and comparative analysis. Wiley interdisciplinary reviews. Syst Biol Med 1:390-399. https://doi. org/10.1002/wsbm.44
Soman C, Lingappa A (2015) Robinow syndrome: a rare case report and review of literature. Int J Clin Pediatr Dent 8:149-152. https ://doi.org/10.5005/jp-journals-10005-1303

Stockard CR (1941) The genetic and endocrinic basis for differences in form and behavior: as elucidated by studies of contrasted pure-line dog breeds and their hybrids. The Wistar Institute of Anatomy and Biology, Philadelphia

Waldo K, Miyagawa-Tomita S, Kumiski D, Kirby ML (1998) Cardiac neural crest cells provide new insight into septation of the cardiac outflow tract: aortic sac to ventricular septal closure. Dev Biol 196:129-144. https://doi.org/10.1006/dbio.1998.8860

White JJ, Mazzeu JF, Coban-Akdemir Z, Bayram Y, Bahrambeigi V, Hoischen A, van Bon BWM, Gezdirici A, Gulec EY, Ramond F, Touraine R, Thevenon J, Shinawi M, Beaver E, Heeley J, HooverFong J, Durmaz CD, Karabulut HG, Marzioglu-Ozdemir E, Cayir A, Duz MB, Seven M, Price S, Ferreira BM, Vianna-Morgante AM, Ellard S, Parrish A, Stals K, Flores-Daboub J, Jhangiani SN, Gibbs RA, Brunner HG, Sutton VR, Lupski JR, Carvalho CMB (2018) WNT signaling perturbations underlie the genetic heterogeneity of Robinow syndrome. Am J Hum Genet 102:27-43. https ://doi.org/10.1016/j.ajhg.2017.10.002

Wickham H (2016) ggplot2: elegant graphics for data analysis. Springer International Publishing

Wickham H, François R, Henry L, Müller K (2019) dplyr: a Grammar of Data Manipulation. R package version 0.8.3. https://CRAN.Rproject.org/package $=$ dplyr

Xing Y, Cheng X, Li Y, Zhang C, Saquet A, Liu Y, Shao M, Shi D (2018) Mutational analysis of dishevelled genes in zebrafish reveals distinct functions in embryonic patterning and gastrulation cell movements. PLoS Genet 14:e1007551. https://doi. org/10.1371/journal.pgen.1007551

Publisher's Note Springer Nature remains neutral with regard to jurisdictional claims in published maps and institutional affiliations. 